\title{
Studies of the Effect of Combined Spectrum LED Phytoradiators on the Growth and Development of Cucumber Plants
}

\author{
${ }^{* 1}$ Olga E. Zheleznikova, ${ }^{2}$ Aleksandr E. Kurshev, ${ }^{3}$ Sergei D. Bogatyrev \\ ${ }^{1}$ Federal State Budgetary Educational Institution of Higher Education "National Research Ogarev Mordovia \\ State University" \\ ${ }^{2,3}$ OJSC Ardatov Lighting Engineering Plant \\ Email: sarstf@mail.ru,a.kurshev@mail.ru,s.bogatyrev@mail.ru
}

Received: 07th February 2020, Accepted: 02nd March 2020, Published: 30th April 2020

\begin{abstract}
The article presents the results of photobiological studies aimed at determining the preferred requirements for the spectrum of combined spectrum LED phytoradiators for the light culture of cucumber plants, variety Liliput F1. The studies were conducted in the artificial climate laboratory of FSBEI HE N.P. Ogarev Moscow State University, using an experimental research hydroponic plant. The results are presented and the effect of the spectral compositions of phytoradiators based on LEDs on the growth and development of Liliput F1 cucumber plants is estimated. The studies (the first vegetation) made it possible to preliminarily identify the most favorable radiation spectrum, which provides the best biometric characteristics and productivity of plants of Liliput F1 cucumbers.
\end{abstract}

Keywords

LED, Phytoradiator, Radiation Spectrum, Light Culture, Cucumber, Photobiological Research

\section{Introduction}

In modern conditions, the intensive development of LED technologies and the reduction of their cost contributes to the active introduction of highly efficient phytoradiators and the development of technological lighting in the structures of the agricultural complex. Currently, light emitting diodes (LEDs) provide an opportunity to create phytoradiators with the spectrum necessary for a particular culture. LED phytoradiators as quasimonochromatic emitters with the primary ranges of the photosynthetically active radiation (PAR), which determine the formation of biomass, morphogenesis and plant metabolism, promote photobiological studies in the modern world [1 - 5].

Recent studies [6] show that the reaction of plants (their productivity) nonadditively depends on the spectral composition and irradiation, and the search for universal spectra of effects on plant productivity is unpromising. That is, there is no alternative to experimental methods for optimizing parameters for plant photoculture.

Under import phase-out of greenhouse agricultural products grown on an industrial scale and in subsidiary and farms, the development and creation of highly efficient phytoradiators of various capacities based on energyefficient LEDs with an optimal phytoradiation spectrum for such vegetable crops as tomatoes and cucumbers in various vegetation periods is a relevant task [7].

LED phytoradiators enhance the efficiency of absorption of light energy by cultivated plants, which will reduce the length of the growing season before fruiting, the productivity of the plants themselves, and also the commercial quality of the products.

\section{Main Part}

The objective of this work is to investigate the effect of combined spectrum LED phytoradiators on the growth and development of Liliput F1 cucumbers.

Experimental studies were conducted on early-ripening cucumbers, variety Liliput F1, which is distinguished by high palatability, versatility of use, high yield, and resistance to many diseases. Liliput F1 cucumbers are distinguished by average branching and a tendency to form lateral determinant shoots; the bush develops independently. Fruits have a cylindrical shape 7-9 cm long, weighing 80-90 g.

The studies were carried out in the artificial climate laboratory of FSBEI HE N.P. Ogarev Moscow State University at an experimental research hydroponic plant (ERHP) with combined-spectrum LED phytoradiators, the principal difference of which is the use of LED compositions based on white and color LEDs, with changeable spectral characteristics over a wide range of photosynthetically active radiation (PAR). Phytoradiators are manufactured by OJSC “Ardatov Lighting Engineering Plant”.

ERHP consists of three sections, separated by opaque screens with common temperature and humidity control sections for the given ranges. Figure 1 shows one of the three sections of the ERHP with a process area of 0.91 $\mathrm{m}^{2}$. During the experiment, the same level of photon irradiation of $250 \pm 10 \mu \mathrm{mol} / \mathrm{s} \cdot \mathrm{m}^{2}$ above the tops of plants was maintained due to a change in the height of the suspension of phytoradiators. 


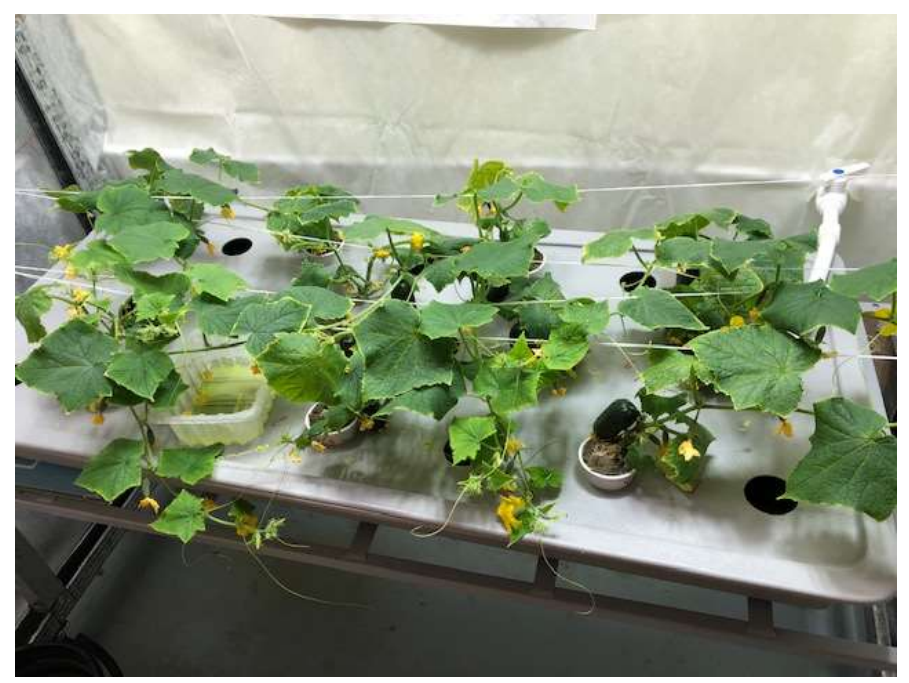

Figure 1: Experimental Research Hydroponic Plant

The first section contains two phytoradiators (PR1) with high-pressure gas discharge sodium arch lamp (the radiation spectrum is shown in Figure 2).

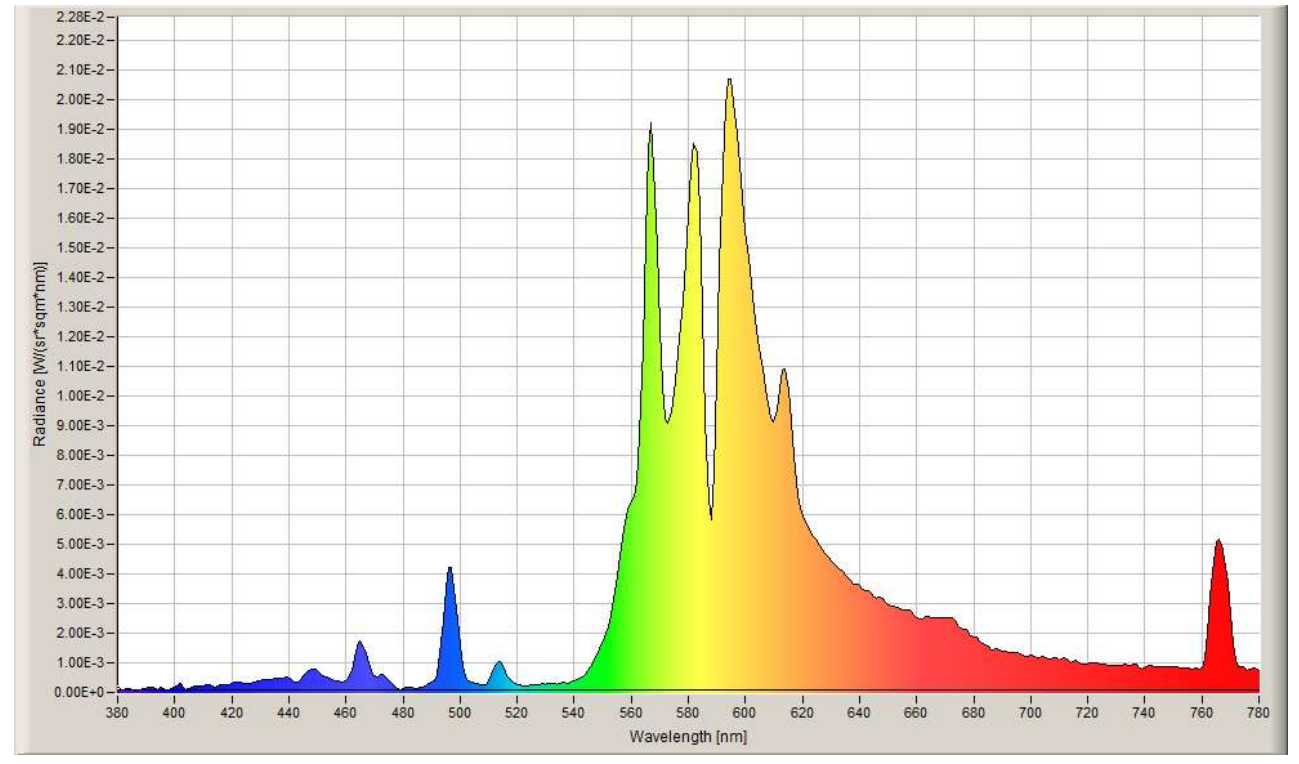

Figure 2: PR1 Radiation Spectrum

The cucumber plants in the second section were irradiated with two phytoradiators (PR2) with GW CSSRM2.PM type LEDs with $\mathrm{T}_{\mathrm{col}}=5000 \mathrm{~K}$ and FM-P3528WNS-460W-R80 with full-spectrum $\mathrm{T}_{\mathrm{col}}=6500 \mathrm{~K}$ and type 2TO3X5FXX000301 and 2TO3X5EXX000301 type LEDs with length $660 \mathrm{~nm}$ and $730 \mathrm{~nm}$, respectively (Figure $3)$.

The third section of the ERHP contains phytoradiators (PR3) with GW CSSRM2.PM type LEDs with a continuous wavelength spectrum and $\mathrm{T}_{\mathrm{col}}=5000 \mathrm{~K}$ and $2 \mathrm{TO} 3 \mathrm{X} 5 \mathrm{FXX} 000301$ type LEDs with a peak wavelength of $660 \mathrm{~nm}$. The emission spectrum of phytoradiator PR3 is shown in Figure 4.

The spectral characteristics of phytoradiators were measured with a Specbos 1211 spectroradiometer (Jeti, Germany), and the irradiation level with a MK 350S spectrometer (UPRtek, Taiwan). The uneven distribution of the irradiation of the process zone in all sections did not exceed $15 \%$.

The experiments (the first vegetation) were carried out at a constant photoperiod of 16 hours and air temperature of $+22-+24^{\circ} \mathrm{C}$. Mineral wool was used as a substrate, a nutrient solution was prepared with the addition of mineral fertilizers: $\mathrm{Ca}\left(\mathrm{NO}_{3}\right)_{2}, \mathrm{NH}_{4} \mathrm{NO}_{3}, \mathrm{KH}_{2} \mathrm{PO}_{4}, \mathrm{~K}_{2} \mathrm{SO}_{4}, \mathrm{MgSO}_{4}, \mathrm{KNO}_{3}$ and Mikrovit complex fertilizer. 


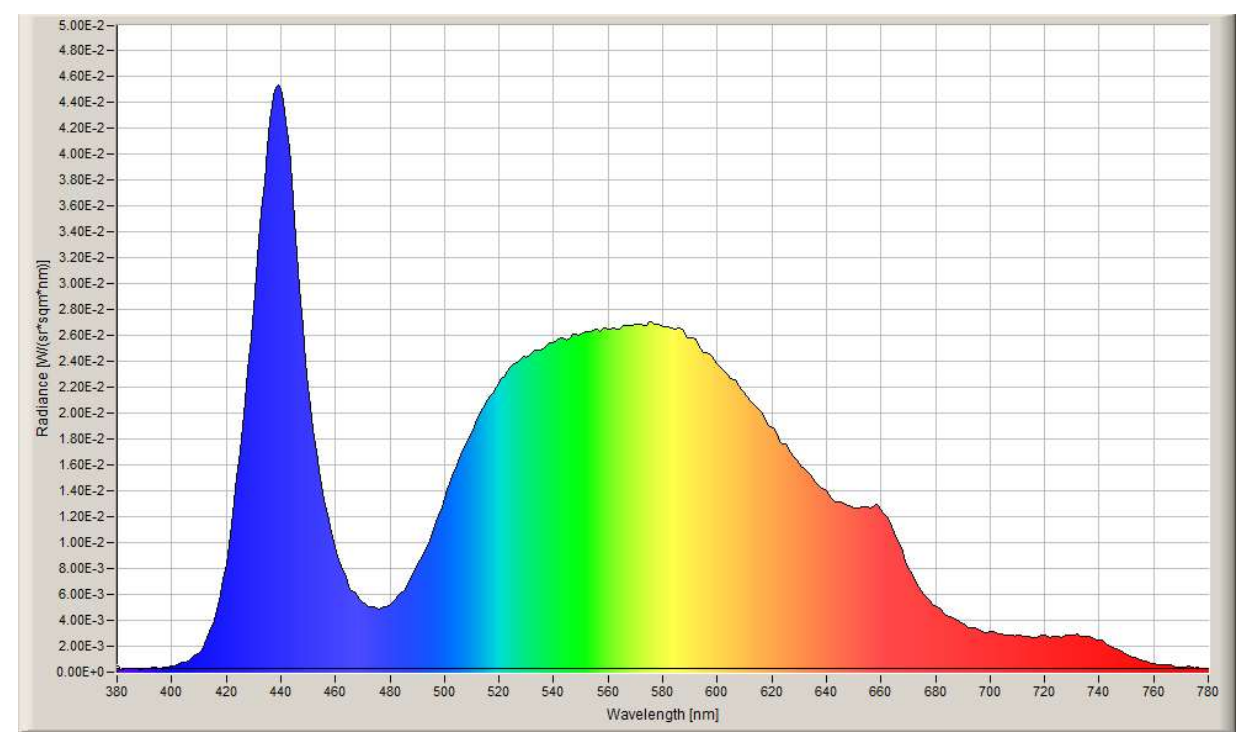

Figure 3: PR2 Radiation Spectrum

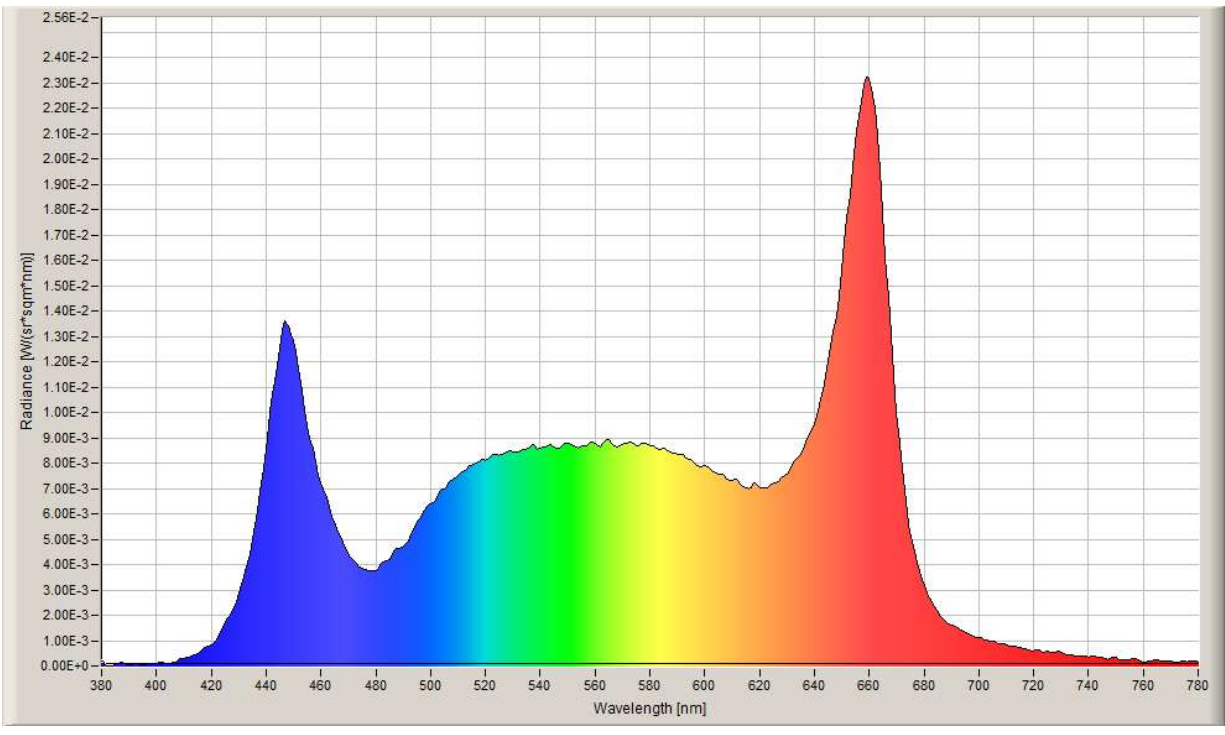

Figure 4: PR3 Radiation Spectrum

The concentration of the nutrient solution of fertilizers was maintained within $\mathrm{E}_{\mathrm{c}}=1.8-2.3 \mathrm{mS} / \mathrm{cm}, \mathrm{PH}=5.5-$ 5.8 , humidity - $70 \% \mathrm{SH}$.

Phenological measurements and observations of plant growth and development were carried out every 5 days. The height of the plants, the area of the largest leaf, the number of leaves, the diameter of the stem, the number of runners, etc. were recorded. Regular observations of seedlings of cucumbers were started at the age of 15 days. Table 1 presents the dynamics of plant growth of Liliput F1 cucumbers.

Table 1: Dynamics of Plant Growth of Liliput F1 Cucumbers

\begin{tabular}{|c|c|c|c|c|c|c|}
\hline \multirow{2}{*}{$\begin{array}{c}\text { Irradiation } \\
\text { variants }\end{array}$} & \multicolumn{5}{|c|}{ Cucumber height, cm } \\
\cline { 2 - 7 } & 25 & 30 & 35 & 40 & 45 & 50 \\
\cline { 2 - 7 } & $20.3 \pm 1.4$ & $38.7 \pm 1.9$ & $48.6 \pm 2.2$ & $52.6 \pm 2.4$ & $53.3 \pm 2.0$ & $58.6 \pm 2.1$ \\
\hline PR1 & $19.3 \pm 1.3$ & $46.3 \pm 1.7$ & $58.6 \pm 1.6$ & $62.3 \pm 1.8$ & $66.3 \pm 1.5$ & $68.6 \pm 2.0$ \\
\hline PR2 & $13.6 \pm 1.2$ & $34.3 \pm 1.4$ & $46.3 \pm 1.9$ & $51.6 \pm 1.8$ & $54.3 \pm 1.9$ & $59.3 \pm 1.9$ \\
\hline PR3 &
\end{tabular}

The analysis of the results of Table 1 allowed us to conclude as follows: from the very beginning of irradiation, we observed a difference in the size and quality of cucumber seedlings for various irradiation variants. Cucumber plants in the time range of 25-35 days under PR3 significantly lagged in growth - in relation to seedlings under 
PR1 by 33\% - 4.7\%, under PR2 - by $29.5 \%$ - 20.9\%. By the age of 40-45 days, the situation changed: the height of cucumber seedlings under PR3 even slightly (on average by 1.5\%) exceeded the height of plants under PR1. Biometric indicators of Liliput F1 cucumbers are shown in Table 2.

On day 50, the height of the cucumber plants under PR2 exceeded the height of plants under PR1 and PR3 by $14.5 \%$ and $13.6 \%$, respectively, and the wet weight was exceeded by $4.2 \%$ and $9.4 \%$, respectively. Therefore, the spectral composition of the irradiation has less effect on the productivity of photosynthesis than on its growth, which is also noted in [8].

Table 2: Biometric Indicators of Liliput F1 Cucumbers

\begin{tabular}{|c|c|c|c|}
\hline \multirow{2}{*}{ Parameter } & \multicolumn{3}{|c|}{ Irradiation variants } \\
\cline { 2 - 4 } & PR1 & PR2 & PR3 \\
\hline Cucumber height, cm & $58.66 \pm 6.2$ & $68.66 \pm 7.1$ & $59.3 \pm 6.3$ \\
\hline Number of leaves, pc. & $16.0 \pm 1.3$ & $18.0 \pm 1.2$ & $16.7 \pm 1.1$ \\
\hline The largest leaf area, cm ${ }^{2}$ & $120.0 \pm 7.1$ & $121.2 \pm 7.4$ & $43.5 \pm 2.5$ \\
\hline Raw mass, g & $46.0 \pm 2.7$ & $48.0 \pm 2.8$ & 6.9 \\
\hline
\end{tabular}

The chlorophyll content in the leaves was estimated using a UV-Mini-1240 spectrophotometer (Table 3).

Table 3: The Chlorophyll Content in the Leaves of 50-Day-Old Liliput F1 Cucumbers

\begin{tabular}{|c|c|c|c|c|}
\hline \multirow[t]{2}{*}{ Irradiation variants } & \multicolumn{3}{|c|}{ Pigment concentration, mg/l } & \multirow{2}{*}{$\begin{array}{l}\text { Pigment content } \\
\text { per raw mass, } \mathrm{mg} / \mathrm{g}\end{array}$} \\
\hline & Chlorophyll a & Chlorophyll b & $\mathrm{a}+\mathrm{b}$ amount & \\
\hline PR1 & $17.79 \pm 0.7$ & $14.58 \pm 0.4$ & $25.7 \pm 1.1$ & $1.71 \pm 0.1$ \\
\hline PR2 & $18.86 \pm 0.8$ & $18.89 \pm 0.5$ & $33.6 \pm 1.0$ & $2.24 \pm 0.2$ \\
\hline PR3 & $6.53 \pm 0.3$ & $3.46 \pm 0.3$ & $10.0 \pm 0.9$ & $0.67 \pm 0.1$ \\
\hline
\end{tabular}

The leaves of cucumber plants under PR2 were dark green, which is explained by the increased content of chlorophyll. The concentration of chlorophylls a and $\mathrm{b}$ in the leaves of cucumber plants under PR2 was 1.3 times higher than under PR1 and 3.36 times higher than under PR3.

\section{Summary}

The conducted photobiological studies (the first vegetation) made it possible to preliminarily identify the most favorable emission spectrum, which provides the best biometric characteristics and productivity of plants of Liliput F1 cucumbers grown with the use of light culture technology, which can be explained by the presence of radiation with a wavelength of $\lambda=730 \mathrm{~nm}$ in the PR2 spectrum, that is, radiation outside the PAR, which is not used and does not exert an energetic effect on plants, but, by exciting the active form of the plant F730 pigment phytochrome regulates photomorphogenesis plants. The influence of the spectral range $(700-780 \mathrm{~nm}) \mathrm{on}$ the growth and development of plants is currently being studied, which makes its presence desirable in the radiation spectrum of phytoradiator $[9,10]$.

\section{Conclusions}

The conducted studies will make it possible to organize the production of unique LED phytoradiators at OJSC Ardatov Lighting Engineering Plant, the spectral radiation flux density of which considers the "preferences" of such vegetable crops as cucumbers during various growing periods.

\section{References}

1. Prikupets L.B., 2017. Technological lighting in agroindustry of Russia. Lighting engineering, 6: 6-14.

2. Kurshev, A.E., Bogatyrev, S.D., Zheleznikova, O.E., Sinitsyna, L.V., 2019. Highly efficient phytoradiator development for plant photoculture based on combined spectrum. International Journal of Innovative Technology and Exploring Engineering, 8 (3): 392-394.

3. Pchelin V.M., Makarova I.E., 2018. Assessment of the status and development prospects of irradiation systems in modern greenhouse complexes. Lighting engineering, 1: 23-27.

4. Lin, K. H., Huang, M. Y., Hsu, M. H., Yang, Z. W., Yang, C. M., 2013. The effects of red, blue and white lightemitting diodes on the growth, development, and edible quality of hydroponically grown lettuce (Lactuca sativa L. var. capitata). Scientia Horticulturae, 150: 86-91.

5. Nanya, K., Ishigami, Y., Hikosaka, S., Goto, E., 2012. Effects of blue and red light on stem elongation and of tomato seedlings. Acta Horticulturae, 956: 261 - 266.

6. Prikupets L.B., Boos G.V., Terekhov V.G., Tarakanov I.G., 2018. Investigation of the effect of radiation in different PAR ranges on the productivity and biochemical composition of the biomass of green salad crops. Lighting engineering, 5: $6-12$.

7. Smallwood, P., 2017. Tracking the Horticultural SSL Market and Technology. Horticultural Lighting Conference: $80-86$. 
8. Rakutko S.A., Rakutko E.N., Vaskin A.N., 2016. The effect of the ratio of red and long-red radiation on the growth and development of tomato seedlings (SOLANUM LYCOPERSICUM). International Journal of Applied and Basic Research, 8: 136-140.

9. Terekhov V.G., 2019. The irradiation system for the City-Farm automated multi-tiered phyto-plant. Lighting engineering, 5: 59 - 63 .

10. Bugbee, B., 2016. Towards an optimal spectral quality for plant growth and development: The importance of radiation capture. ActaHortic, 1134(1): 1-12. 